\title{
Frontiers in cancer endocrinology
}

\section{Annamaria Colao and Gaetano Lombardi*}

Department of Molecular and Clinical Endocrinology and Oncology, Federico I/ University of Naples, Naples, Italy ${ }^{*}$ Correspondence: rpivone@tin.it

The "Classical Endocrinology" is the fascinating world of endocrine glands, their specific hormones and their diseases. This encapsulates the integration of developmental events such as the proliferation, growth, and differentiation of tissues and organs and the coordination of many physiological functions, such as metabolism and reproduction, by chemical substances synthesized and secreted by specialized cells. A modern view of Endocrinology requires, in our opinion, a more detailed sight in the field not only of endocrine glands and their diseases, but also of the multitude of organs and cells involved, influenced and eventually controlled by large intricate pathways of signaling activated by a very heterogeneous group of substances targeting a wide spectrum of receptors and cells throughout the body. Therefore, today "Cancer Endocrinology" necessarily means focusing on all endocrine tumors from classical ones, such as pituitary, thyroid, parathyroid, adrenal, and neuroendocrine tumors, to types of cancer in which endocrine hormones seem to play a relevant role such as breast, prostate, ovaries, colon, and liver tumors and probably many others. As such Cancer Endocrinology should now be of particular interest for experimental, preclinical, and clinical researchers. Cancer Endocrinology now encompases the following issues: identification of a definitive role for new laboratory tests and radiological techniques in diagnosis; identification of specific molecular patterns of tumorigenesis, which could allow development of new directions in the field of pharmacotherapy research; combined treatment with conventional treatment options and new molecules in preclinical evaluation; synergy between chemotherapy and other anticancer modalities, including radiotherapy, immunotherapy, and gene therapy; discovery of new biomarkers to predict response or resistance to drug treatment or to guide the follow-up of treated patients.

Examples of developments in cancer endocrinology include the role of somatostatin (SSTR) and dopamine receptors
(DR) as molecular targets for the treatment of patients with pituitary adenomas and neuroendocrine tumors: this has received great attention and is leading to innovative new therapies. The evidence of expression of subtypes of SSTR and DR, as well as of co-expression of these receptors in tumor cells, has increased the development of new experimental drugs, including novel analogs, binding to multiple SSTR subtypes, as well as hybrid somatostatin-dopamine compounds. These advances have opened new perspectives for the medical treatment of pituitary and neuroendocrine tumors poorly responsive to conventional therapies and probably also for the treatment of other tumor types. Pasireotide (SOM230), a novel somatostatin analog with a peculiar receptor binding that makes this analog the closest to native somatostatin, is under detailed investigation in Cushing's disease, so far an orphan disease for medical therapy. Everolimus, an m-TOR inhibitor, indicated for treatment of renal carcinoma, has also been demonstrated to have some efficacy for patients with neuroendocrine tumors supplementing the medical armamentarium for management of these patients. Nonetheless, today management of aggressive pituitary adenomas still remains a challenging clinical problem: new potential therapies such as gene therapy, temozolomide, or a combination of target therapies are foreseen as future strategies for these patients.

Another important endocrine axis to be considered in carcinogenesis are represented by growth factors such as the insulin/insulin-like growth factor-I (IGF I). The IGF system mediates growth, differentiation, and developmental processes. Clinical conditions associated with high levels of insulin (non-insulin-dependent diabetes mellitus and hypertriglyceridemia) and IGF-I (acromegaly) are related to increased risk of neoplasms, and increased circulating concentrations of insulin and IGF-I are related to a higher risk of colonic neoplasia. Deregulation of IGF system expression and action is linked to different diseases, ranging from growth deficits to cancer development. Targeting of the IGF axis has emerged in recent years as a promising therapeutic approach in cancer and other conditions. Rational use of IGF-I-induced gene signatures may help to identify patients who might benefit from IGF axis-directed therapeutic modalities. IGF-I-induced gene expression in primary breast and lung fibroblasts accurately predict outcomes in breast and lung cancer patients. Further studies to investigate the role of IGF-pathway on colorectal, prostatic, and breast and lung cancer are required.

Classically modulation of gonadal steroids is used as adjuvant treatment in patients with breast and prostate cancers. However, hypogonadism (as a consequence of ovarian and testicular blockade) causes insulin resistance so that patients would probably acquire a secondary cause of tumor relapse as discussed above. No data are currently available on this matter but it is expected that use of insulin-sensitizers (currently under investigation) would modify the natural history of breast and prostate cancer patients treated with the estrogens or androgens blockade.

A separate field of investigation relates to the so-called "neuroendocrine degeneration" of some cancers, particularly the prostate. The clinical significance and possible treatment approach of tumors with such a phenotype is still far from being elucidated.

Lastly, considerable preclinical and epidemiologic data suggest that vitamin $\mathrm{D}$ may play a role in the pathogenesis, progression, and therapy for cancer. The relevance of vitamin D receptor (VDR) gene polymorphisms for various types of cancer has been widely investigated. It has been hypothesized that VDR polymorphisms may influence both the risk of cancer occurrence and prognosis. However, studies investigating the associations between specific VDR polymorphisms and cancer often show controversial results. Data indicating an association of VDR polymorphisms and cancer risk are relevant for breast cancer, prostate cancer, and malignant melanoma. Higher risk of cancer has 
been linked with lower serum vitamin D levels. Unfortunately, very limited data are available to indicate whether or not giving vitamin D supplements reduces the risk of cancer. Many preclinical studies indicate that exposing cancer cells or vascular endothelial cells derived from tumors, to high concentrations of vitamin D could reduce progression through the cell cycle, induce apoptosis and slow or stop the growth of tumors in vivo. Despite these observations there are no data indicating that one type of cancer is more or less susceptible to the effects of vitamin D. Vitamin $\mathrm{D}$ also potentiates the antitumor activity of a number of types of cytotoxic anticancer agents examined in preclinical models. Vitamin D analogs initiate signaling through a number of important pathways, but the pathway(s) essential to the antitumor activities of vitamin D are unclear. In view of the substantial preclinical and epidemiologic data supporting the potential role of vitamin D in cancer, further studies to evaluate the impact of vitamin D replacement on the frequency of cancer and the impact of an appropriate dose and schedule of treatment are indicated.

The vitamin $\mathrm{D}$ and insulin axes are also related to the wider argument of the nutritional background of cancer, tightly linked to the hormonal regulation of cell differentiation.

In conclusion, the endocrine system seems to be a key to open the full understanding of tumoral development. A multifaceted approach involving genetics, cellular and molecular biology, pathophysiology, and oncology would allow a better knowledge of endocrine tumor pathogenesis and eventually to the development of new therapeutic strategies in all the field of the "Cancer Endocrinology."

Received: 08 December 2010; accepted: 25 February 2011; published online: 11 March 2011.

Citation: Colao A and Lombardi $G$ (2011) Frontiers in Cancer Endocrinology. Front. Endocrin. 2:7. doi: 10.3389/ fendo.2011.00007

This article was submitted to Frontiers in cancer endocrinology, a specialty of Frontiers in Endocrinology.

Copyright $\odot 2011$ Colao and Lombardi. This is an openaccess article subject to an exclusive license agreement between the authors and Frontiers Media SA, which permits unrestricted use, distribution, and reproduction in any medium, provided the original authors and source are credited. 